\title{
EGFR inhibition attenuates diabetic nephropathy through decreasing ROS and endoplasmic reticulum stress
}

\author{
Zheng $\mathrm{Xu}^{1, *}$, Yunjie Zhao ${ }^{1, *}$, Peng Zhong ${ }^{1}$, Jingying Wang ${ }^{1}$, Qiaoyou Weng ${ }^{1,3}$, \\ Yuanyuan Qian', Jibo Han ${ }^{1}$, Chunpeng Zou ${ }^{2}$, Guang Liang ${ }^{1}$ \\ ${ }^{1}$ Chemical Biology Research Center, School of Pharmaceutical Sciences, Wenzhou Medical University, Wenzhou, Zhejiang, \\ 325035, China \\ ${ }^{2}$ Department of Ultrasonography, the Second Affiliated Hospital and Yuying Children's Hospital of Wenzhou Medical University, \\ Wenzhou, Zhejiang, 325000, China \\ ${ }^{3}$ Department of Interventional Radiology, the Fifth Affiliated Hospital of Wenzhou Medical University, Lishui, Zhejiang, \\ 323000, China \\ *These authors contribute equally to this work
}

Correspondence to: Guang Liang, email: wzmcliangguang@163.com Chunpeng Zou, email: chpzou@126.com

Keywords: diabetic nephropathy, epidermal growth factor receptor, inhibitor, ER stress, oxidative stress

Received: December 06, 2016

Accepted: February 23, 2017

Published: March 06, 2017

Copyright: Xu et al. This is an open-access article distributed under the terms of the Creative Commons Attribution License (CC-BY), which permits unrestricted use, distribution, and reproduction in any medium, provided the original author and source are credited.

\section{ABSTRACT}

Diabetic nephropathy (DN) is a progressive kidney disease due to glomerular capillary damage in diabetic patients. Endoplasmic reticulum (ER) stress caused by reactive oxygen species (ROS) is associated with DN progression. Epidermal growth factor receptor (EGFR) mediates oxidative stress and damage of cardiomyocytes in diabetic mice. Here we demonstrated that AG1478, a specific inhibitor of EGFR, blocked EGFR and AKT phosphorylation in diabetic mice. Oxidative stress and ER stress markers were eliminated after AG1478 administration. AG1478 decreased pro-fibrotic genes TGF- $\beta$ and collagen IV. Furthermore, we found that high glucose (HG) induced oxidative stress and ER stress, and subsequently increased ATF4 and CHOP. These changes were eliminated by either AG1478 or ROS scavenger N-acetyl-L-cysteine (NAC) administration. These results were confirmed by knock-down approaches in renal mesangial SV40 cells. However, AG1478, not NAC, reversed HG induced EGFR and AKT phosphorylation. These results suggest that EGFR/AKT/ROS/ER stress signaling plays an essential role in DN development and inhibiting EGFR may serve as a potential therapeutic strategy in diabetic kidney diseases.

\section{INTRODUCTION}

Diabetes mellitus is a major health risk worldwide. The metabolic abnormality and microvascular complications caused by the sustained hyperglycemia lead to diabetic nephropathy (DN) [1]. Diabetic kidney disease is the leading cause of morbidity and end-stage renal disease (ESRD) [2-4]. However, effective clinical interventions for DN have not yet been elucidated. Although great advances have been accomplished in tight glycemic control and blood pressure control $[5,6]$, they cannot stop DN progression.

Many receptor tyrosine kinases (RTKs) are implicated in DN pathogenesis. The epidermal growth factor receptor (EGFR) is an important member of RTKs [7]. After ligand binding, EGFR signaling is triggered by phosphorylation cascades intracellularly. EGFR is widely expressed in glomeruli, proximal tubes and collecting ducts. EGFR phosphorylation is increased in diabetic animals [8], suggesting its activation is involved in diabetic damages. Furthermore, recent studies have demonstrated that pharmacological antagonists of EGFR efficiently block downstream cascades activation in diabetic animals [8]. EGFR is pathogenic in various nephron cell types. Hyperglycemia increases reactive oxygen species (ROS) in diabetic patients. Accumulating evidence indicates that the ROS overproduction contributes to DN pathogenesis. We previously demonstrated that activation of EGFR leads to cardiac damage and remodeling via ROS generation in STZ-induced diabetic mice [9]. Inhibiting EGFR protects cardiomyocytes from oxidative stress by reducing ROS accumulation. Blockage of AKT prevents HG-induced 
ROS generation and cardiomyocyte damage [9]. Thus, inhibiting EGFR activation may also serve as a therapeutic target for DN. In addition, Endoplasmic reticulum (ER) stress, with misfolded and/or unfolded proteins in ER membranes, has been shown to mediate DN development and progression [10]. The crosstalk between ER stress and ROS production is well established in cancer biology, in which increasing ROS induces ER stress to trigger cancer cell apoptosis [11-13]. Interestingly, ERGR signaling plays a key role regulating ER stress and ROS accumulation [14, 15]. However, whether EGFRmediated ER stress and ROS generation contribute to DN progression remains unexplored. Although several mechanisms are involved in the regulation of EGFR, the interactions between EGFR, ROS generation/ER stress are unknown in the progression of DN.

In this study, we investigated the role of EGFR/ $\mathrm{AKT} / \mathrm{ROS} / \mathrm{ER}$ stress pathway in the progression of DN using AG1478, a specific and commercial inhibitor of EGFR. AG1478 inhibited EGFR and AKT activation, ameliorating ROS accumulation and ER stress and preventing diabetes-induced renal injury. Thus blocking EGFR/AKT/ROS/ER stress pathway may serve as a potential therapeutic strategy in preventing diabetic nephropathy.

\section{RESULTS}

\section{AG1478 administration decreased diabetes- induced renal injury}

Streptozotocin (STZ)-induced diabetic mice showed elevated blood glucose level (Supplementary Figure 1A) and the relatively lower body weight (Supplementary Figure 1B). However, AG1478 (20mg/kg) treatment for 8 weeks did not affect blood glucose level or body weight in either T1DM group or AG1478-treated group (Supplementary Figure 1). Renal injury was examined by albumin reagent kit, and renal fibrosis was examined by Sirius Red staining and Masson's trichrome staining, respectively. The diabetic animals induced by STZ injection showed decreased serum albumin, elevated kidney/body weight ratio, histological abnormalities, and renal fibrosis (Figure 1A-1C). Administration of AG1478 significantly improved serum albumin level and decreased the kidney/body weight ratio in diabetic mice (Figure 1A and $1 \mathrm{~B})$. Histological abnormalities and fibrosis in diabetic mice were ameliorated by AG1478 treatment (Figure 1C). Western blot analysis indicated significant reduction in the expression of pro-fibrotic gene TGF- $\beta 1$ and collagen IV in diabetic kidneys (Figure 1D). Real-time qPCR assay also exhibited a remarkable increase in pro-fibrotic genes including collagen-IV and TGF- $\beta 1$ (Figure $1 \mathrm{E}$ and $1 \mathrm{~F}$ ). AG1478 treatment also significantly decreased renal injury and fibrosis markers (Figure $1 \mathrm{~A}-1 \mathrm{~F}$ ). As shown in Figure 2A, apoptosis cells stained in bright green by
TUNEL assay were remarkably increased in diabetic mice. AG1478 treated group significantly reversed diabetesinduced nephrocyte apoptosis. The expression of proapoptotic protein Bax in mouse kidneys was assessed by western blot analysis. As expected, the results indicated that treatment with AG1478 inhibited diabetes-induced Bax upregulation (Figure 2B).

\section{AG1478 attenuated renal EGFR signaling activation in diabetic mice}

The EGFR signaling is activated in early diabetes and plays an important role in kidney hypertrophy and fibrosis. Here we observed that EGFR phosphorylation was up-regulated in diabetic mice, both at cellular levels and total protein levels (Figure 3A, 3B). However, AG1478 treatment dramatically decreased EGFR phosphorylation in diabetic kidneys (Figure 3A, 3B), suggesting that AG1478 eliminated EGFR activation. Since EGFR is known to turn on PI3K/AKT signaling [16], we explored whether AG1478 regulates this major downstream target phosphorylation. We found that AKT was also significantly activated in diabetic kidneys, which was markedly inhibited in AG1478-treated animals (Figure 3C). These data suggested that EGFR and AKT were activated during DN progression, and AKT phosphorylation directly responded to EGFR activation.

\section{AG1478 attenuated diabetes-induced renal oxidative stress and ER stress}

Mounting evidence has established that oxidative stress and ER stress are entwined phenomena, contributing to the diabetes-induced pathological changes. Therefore, we investigated whether oxidative stress and ER stress were involved in the attenuation of diabetic nephropathy after EGFR inhibition. IHC staining analysis showed that both oxidative stress markers (DHE and 3-NT) and ER stress markers (ATF4 and CHOP) were increased in STZinduced diabetic kidneys (Figure 4A, 4B). Significantly, AG1478 administration could eliminate these changes. The results indicated that AG1478 treatment markedly reduced renal oxidative stress (Figure 4A) and inhibited renal ER stress (Figure 4B), suggesting that the protective effects of EGFR blockade may be associated with the inhibition of oxidative stress and ER stress.

\section{AG1478 and NAC inhibited HG-induced ROS generation, ER stress, apoptosis, and fibrosis}

In vivo study has showed that EGFR inhibitor AG1478 can attenuate renal oxidative stress and renal injury in mouse model with type 1 diabetes. An antioxidant, $\mathrm{N}$-acetyl-L-cysteine (NAC) which is well-known to mitigate the increased oxidative stress, was used for in vitro study. To investigate whether the inhibition of EGFR 
activity and ROS affects the aforementioned damage in vitro, mesangial cells cultured in high-glucose medium were pretreated with AG1478 or NAC. As indicated in Figure 5A, renal mesangial SV40 cells endogenously expressed a relative high amount of EGFR and AKT. HG treatment significantly increased the phosphorylation of both EGFR and AKT in SV40 cells, whereas these alterations were reversed by AG1478 pre-treatment. In contrast, NAC pre-treatment had no obvious effects.

Furthermore, we determined the effects of EGFR inhibitor on HG-induced ROS generation and cell damage. As shown in Figure 5B, HG treatment for $1 \mathrm{~h}$ markedly increased ROS generation, which was significantly reduced by pre-treatment with either AG1478 or NAC in SV40 cells, as indicated by DCFH-DA fluorescence staining and flow cytometry. These results indicated that EGFR signaling may be a mediator of $\mathrm{HG}$-induced ROS generation. We further found that AG1478 or NAC administration markedly reduced HG-induced ATF4/ CHOP expression and PERK/eIF $2 \alpha$ phosphorylation in SV40 cells (Figure 5C). We next examined the effects of EGFR inhibitor or ROS scavenger on the renal cell fibrosis and apoptosis in HG-treated SV40 cells (Figure 5D). Elevated protein expression of TGF- $\beta 1$ and Caspase- 3 by HG could be reduced by either AG1478 or NAC. The results indicated that HG could induce the DN phenotypes in SV40 cells via EGFR activation, both EGFR inhibitor and ROS scavenger could prevent the cell damages, suggesting that EGFR and ROS play pivot roles in mediating hyperglycemia-induced renal pathogenesis.
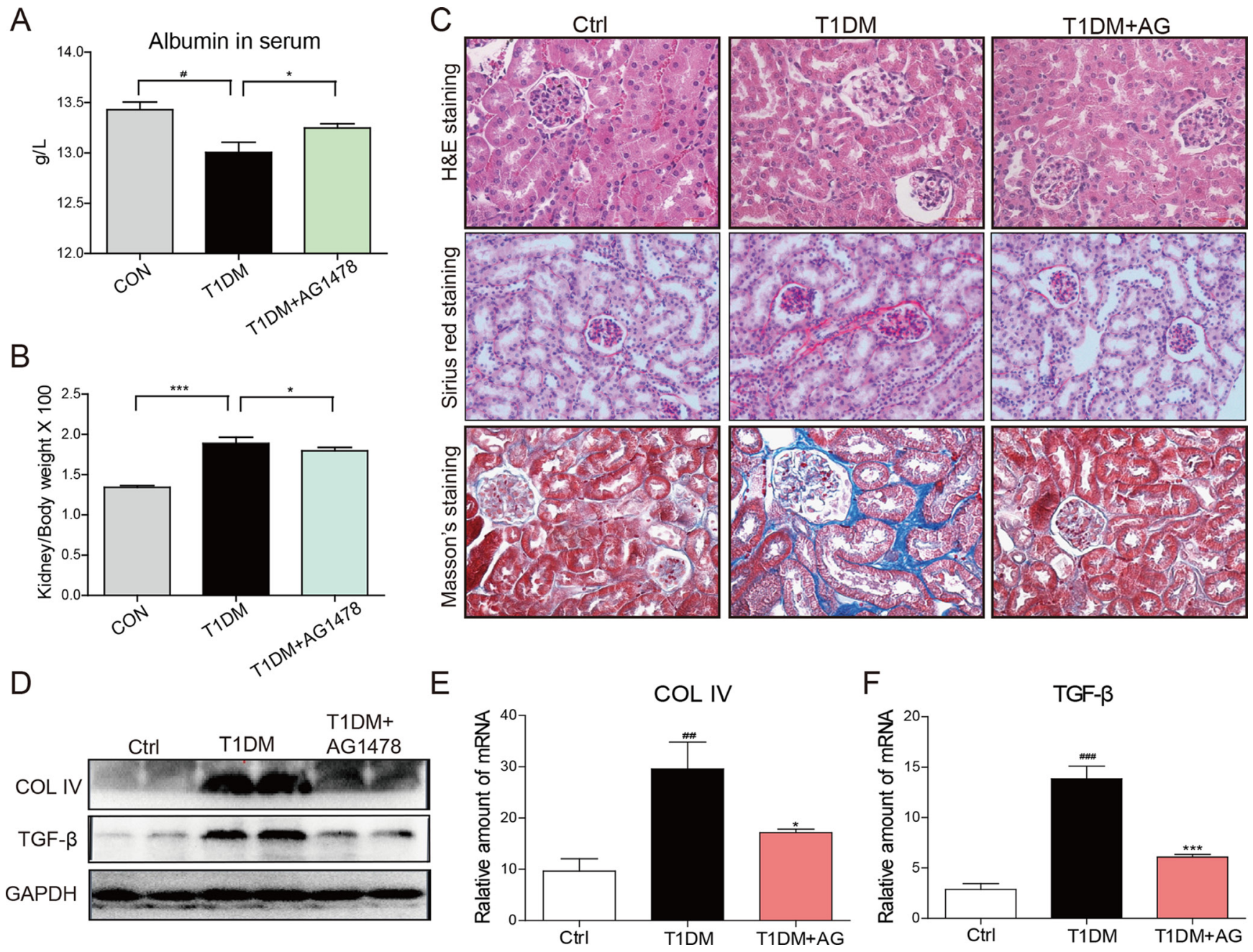

Figure 1: AG1478 attenuate diabetes-induced renal histological abnormalities and fibrosis. The effects of AG1478 on the metabolic profiles of STZ-induced mice: serum albumin (A) and kidney/body weight ratio (B) Serum albumin and kidney/body weight ratio were detected at the time of death. Data are mean \pm S.E.M. (C) Administration of AG1478 significantly improved histologic abnormalities and fibrosis in the formalin-fixed renal tissues. Renal histopathologic analysis was performed using H\&E staining (400×); representative figures of Sirius red staining and Masson trichrome staining $(400 \times)$ on renal tissue. (D) Western blot analysis for Col4 and TGF- $\beta 1$ protein expression in the renal tissue. ( $\mathbf{E}$ and $\mathbf{F}$ ) The mRNA expression of the Col4 and TGF- $\beta 1$ in the renal tissues was measured by real-time qPCR. (Eight mice in each group were used for above analysis; ${ }^{*} P<0.05$, ${ }^{* * *} P<0.001$ versus $\mathrm{DN}$; ${ }^{*} P<0.05$, ${ }^{\#} P<0.01$, ${ }^{\# \#} P<0.001$ versus vehicle control (Ctrl)). 
A
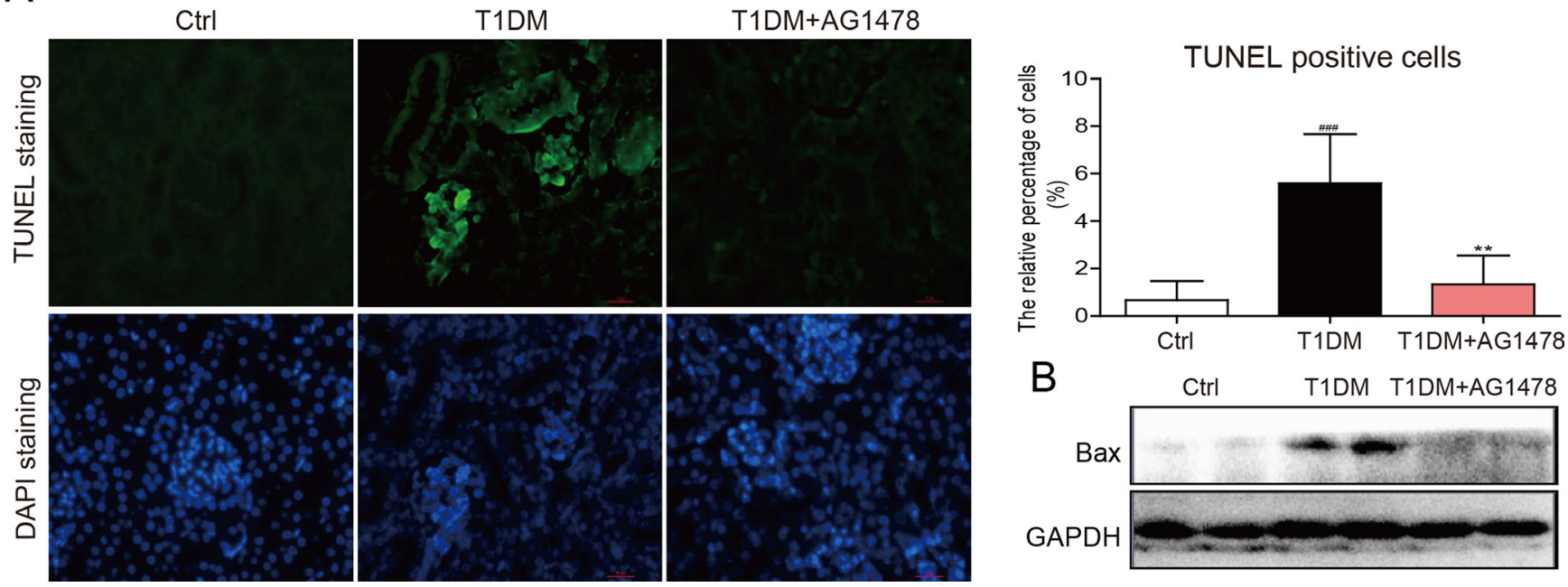

Figure 2: AG1478 mitigate apoptosis in diabetic kidney. (A) Representative images for TUNEL staining in renal tissue sections. Statistic data of TUNEL positive cell was shown, data were presented as mean \pm SDs; (B) Western blot analysis for the protein expression of apoptosis-related proteins Bax in renal tissues. (Eight mice in each group were used for above analysis. ${ }^{* *} P<0.01$ versus DN; \#\# $P<0.001$ versus vehicle control $(\mathrm{Ctrl}))$.

A

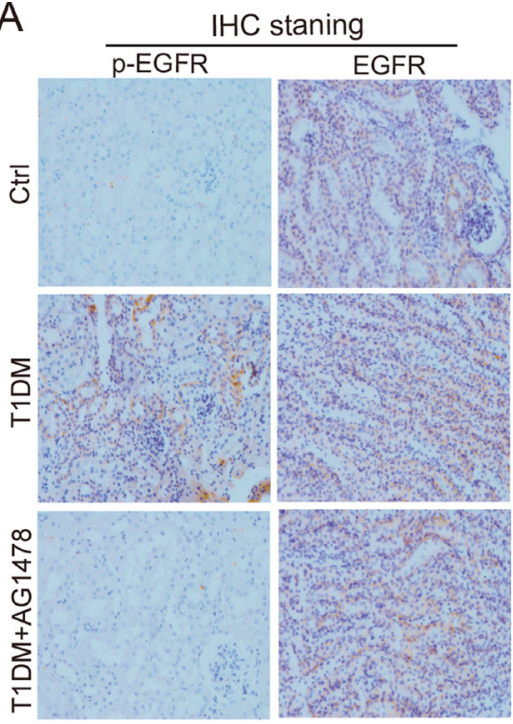

B

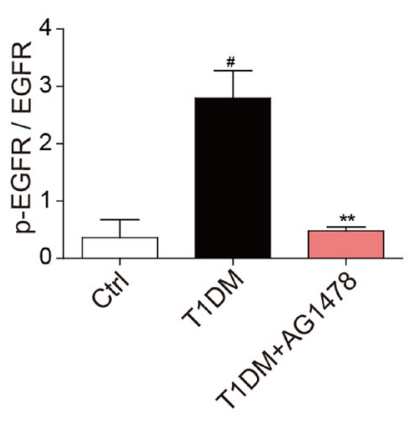

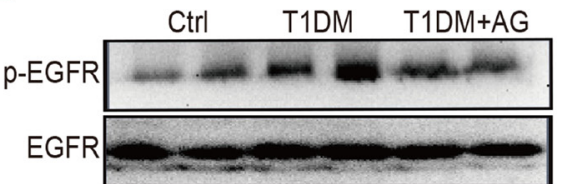

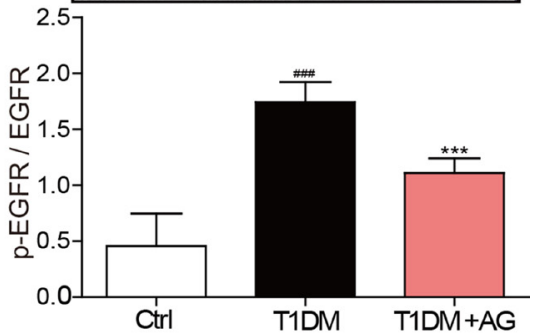

C

Ctrl T1DM T1DM+AG1478
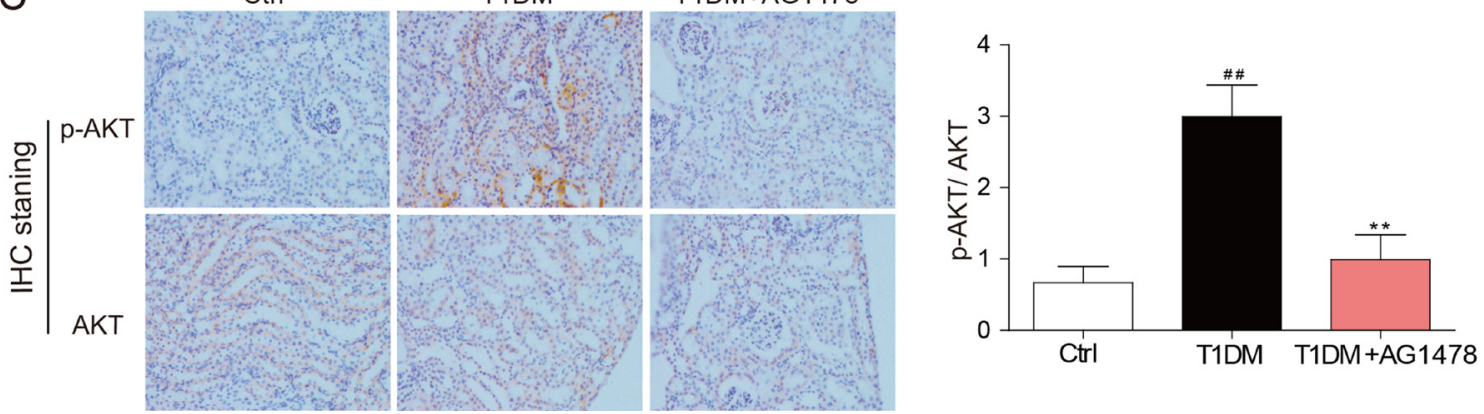

Figure 3: AG1478 attenuate diabetes-induced EGFR signaling activation in diabetic kidney. (A) Representative images for the histochemical staining for p-EGFR and EGFR expression in the formalin-fixed renal tissues (200× magnification). (B) Western blot analysis for the expression of p-EGFR in renal tissue. And statistic figure was shown, data were presented as mean \pm SDs. (C) Representative images for the histochemical staining for p-AKT and AKT expression in the formalin-fixed renal tissues (200× magnification). (Eight mice in each group were used for above analysis. ${ }^{* *} P<0.01,{ }^{* * *} P<0.001$ versus $\mathrm{DN},{ }^{\sharp} P<0.05,{ }^{\#} P<0.01,{ }^{\# \#} P<0.001$ versus vehicle control $(\mathrm{Ctrl})$ ). 


\section{EGFR and AKT siRNA inhibited HG-induced markers for ER stress, apoptosis, and fibrosis in SV40 cells}

In order to avoid the non-specificity of smallmolecular inhibitor AG1478, we used EGFR and AKT siRNA to specifically silence EGFR/AKT expression in SV40 cells. As indicated in Figure 6A, western blot analysis demonstrated that the transfection of EGFR siRNA resulted in a remarkable decrease in EGFR expression in SV40 cells, compared to control scrambled siRNA. Furthermore, as shown in Figure 6B, EGFR siRNA-transfected group markedly inhibited HGinduced AKT phosphorylation, ATF4 and CHOP protein expression, PERK and eIF $2 \alpha$ phosphorylation, TGF- $\beta 1$ protein expression, and Caspase- 3 activation. Figure 6C and 6D shows that inhibition of AKT suppression by siRNA also decreased HG-induced PERK/eIF $2 \alpha$ phosphorylation and ATF4/CHOP/TGF- $\beta 1 /$ Caspase-3 protein expression in SV40 cells. Directly knocking down EGFR using siRNA significantly inhibited the phosphorylation of AKT induced by HG (Figure 6B), confirming that AKT is a downstream mediator of EGFR. In conclusion, knocking down EGFR and AKT prevented the HG-induced ER stress, pro-apoptosis, and fibrosis, indicating that the EGFR/AKT/ROS/ER stress pathway contributes to the hyperglycemia-induced renal cell damages.
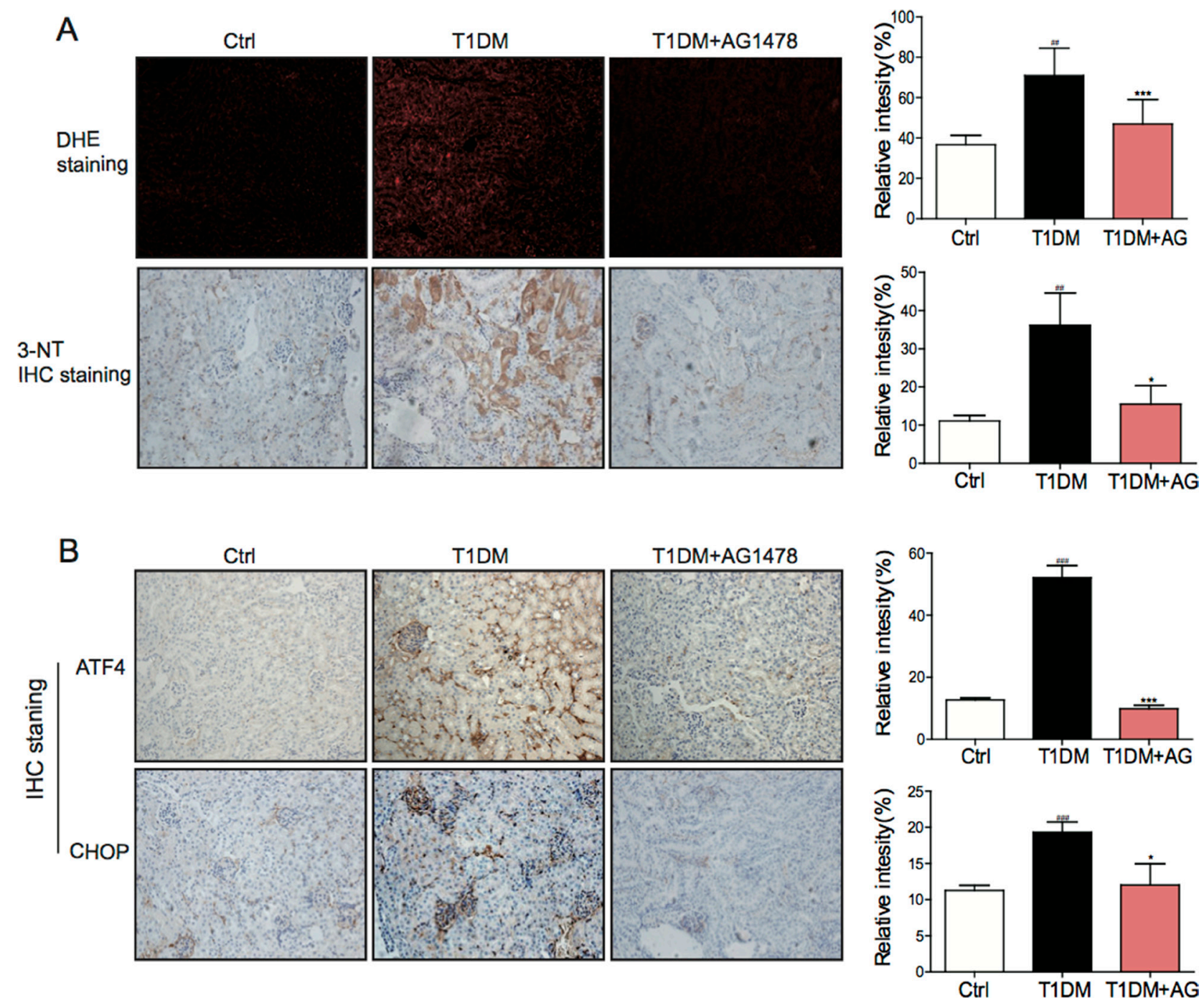

Figure 4: AG1478 attenuate diabetes-induced oxidative stress and endoplasmic reticulum stress. (A) Representative images for DHE staining using the formalin-fixed renal tissues as described in materials and method (200× magnification). Representative images for immunohistochemial staining of 3-NT accumulation using the formalin-fixed renal tissues as described in materials and methods section(200× magnification). And statistic figure was shown, data were presented as mean \pm SDs. (B) Representative images for immunohistochemical staining of ATF4 and CHOP accumulation using the formalin-fixed renal tissues as described in Materials and methods (200× magnification). And statistic figure was shown, data were presented as mean \pm SDs. (Eight mice in each group were used for above analysis. ${ }^{*} P<0.05,{ }^{* * *} P<0.001$ versus $\mathrm{DN} ;{ }^{\# \#} P<0.01,{ }^{\# \#} P<0.001$ versus vehicle control (Ctrl)). 


\section{DISCUSSION}

Our studies demonstrated that renal EGFR phosphorylation stimulates ROS accumulation and ER stress in STZ-induced diabetic animals. Administration of EGFR inhibitor AG1478 attenuated DN, accompanied with EGFR/AKT inactivation and the decreased ROS and inhibited ER stress. Then, we use in vitro study to confirm this pathway in renal mesangial cells. AG1478 blocked HG-induced AKT phosphorylation, ROS production, and

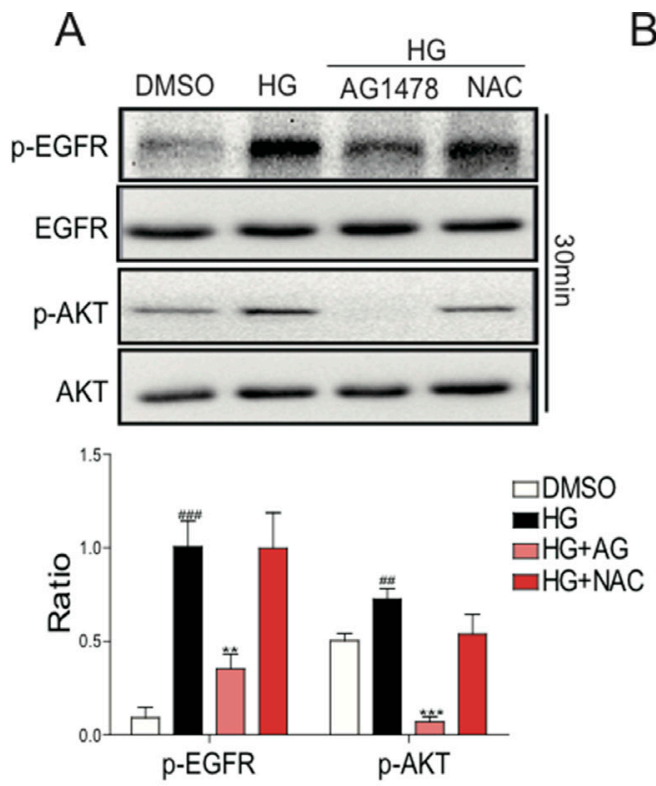

B
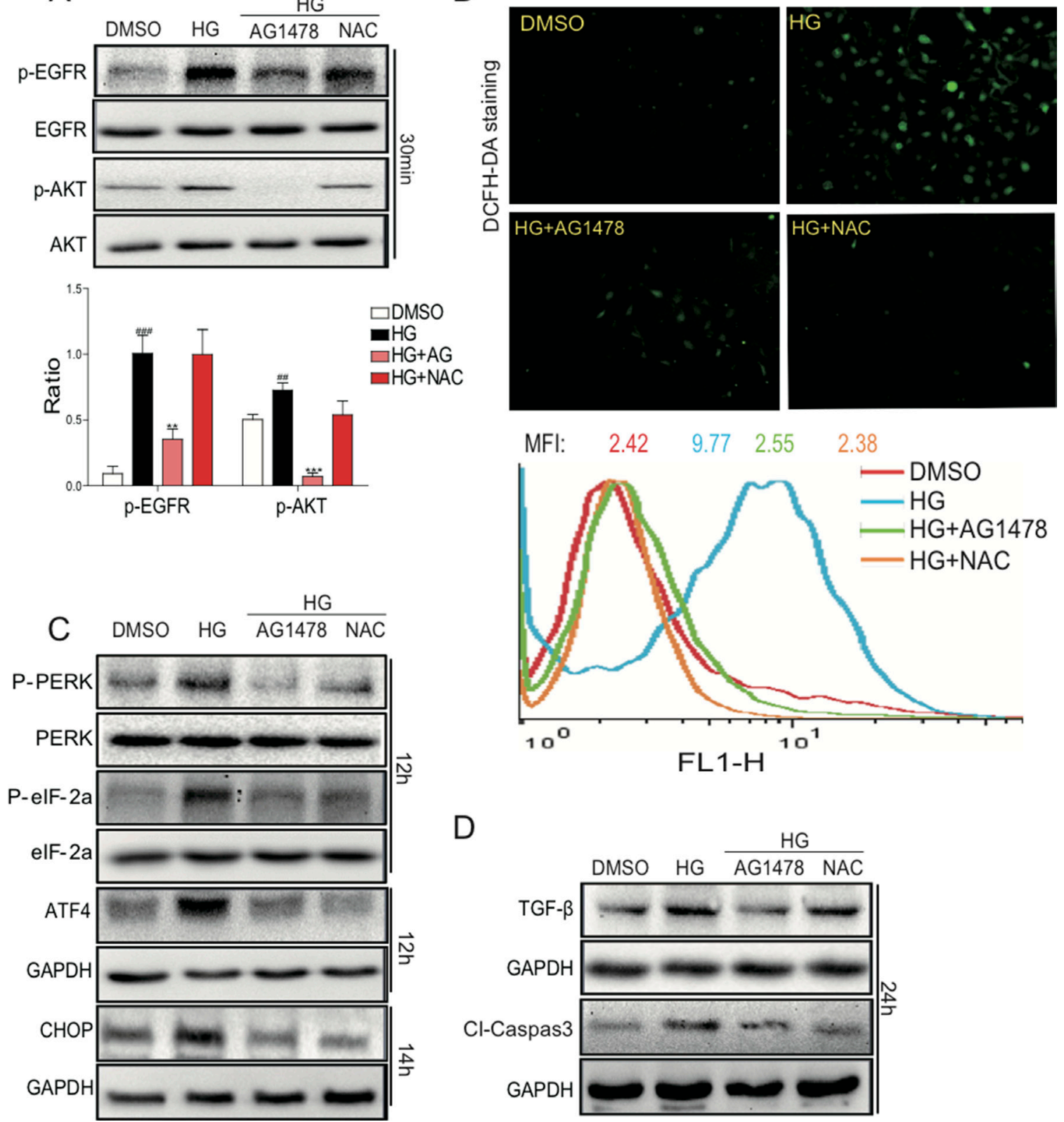

Figure 5: AG1478 and NAC attenuate HG-induced EGFR signaling activation, ROS generation, endoplasmic reticulum stress, cell firosis and apoptosis in SV40 cells. SV40 cells pretreated with AG1478 $(10 \mu \mathrm{M})$ or NAC $(10 \mathrm{mM})$ for $1 \mathrm{~h}$ were incubated with $\mathrm{HG}(33 \mathrm{mM})$ for $1 \mathrm{~h}$. Then cells were lysed and the extracted total proteins were processed for the detection of p-EGFR and p-AKT using Western blot; and statistic figure was shown, data were presented as mean \pm SDs. (Six mice in each group were used for above analysis (A). (B) AG1478 and NAC inhibit high glucose-induced ROS generation. SV40 cells pretreated with AG1478 (10 $\mu$ M) or NAC (10mM) for $1 \mathrm{~h}$ were incubated with HG $(33 \mathrm{mM})$ for $2 \mathrm{~h}$. DCFH-DA probes were loaded and the ROS positive cells were detected using the fluorescence microscope. Also, after loading with the probes, cells were processed to flow cytometry analysis for $\mathrm{O} 2$ level, and mean fluorescence intensity (MFI) value was determined. Data were presented as mean \pm SDs. $\left(n=3\right.$ for each experiment. ${ }^{* *} P<0.01,{ }^{*} * * P<0.001$ versus HG; ${ }^{\prime \prime} P<0.01, \# \#+0.001$ versus DMSO). (C) Western blot analysis for the protein expression of ERS-related proteins ATF4 and CHOP in SV40 cells. (D) Western blot analysis for the fibrosis and apoptosis protein expression of TGF- $\beta 1$ and Cleaved-Caspase 3 in SV40 cells. 
ER stress, while NAC only blocked ROS production and ER stress. To avoid the specificity of AG1478 and to see the role of AKT, we used specific siRNA to silence EGFR and AKT, and similar results were observed. Finally, a clear pathway "from EGFR to AKT, ROS, and ER stress, and finally to kidney fibrosis" has been demonstrated (Figure 7). These results suggested that targeting EGFR could be future therapeutic approach for DN to reduce the physical and psychosocial burdens of diabetes.
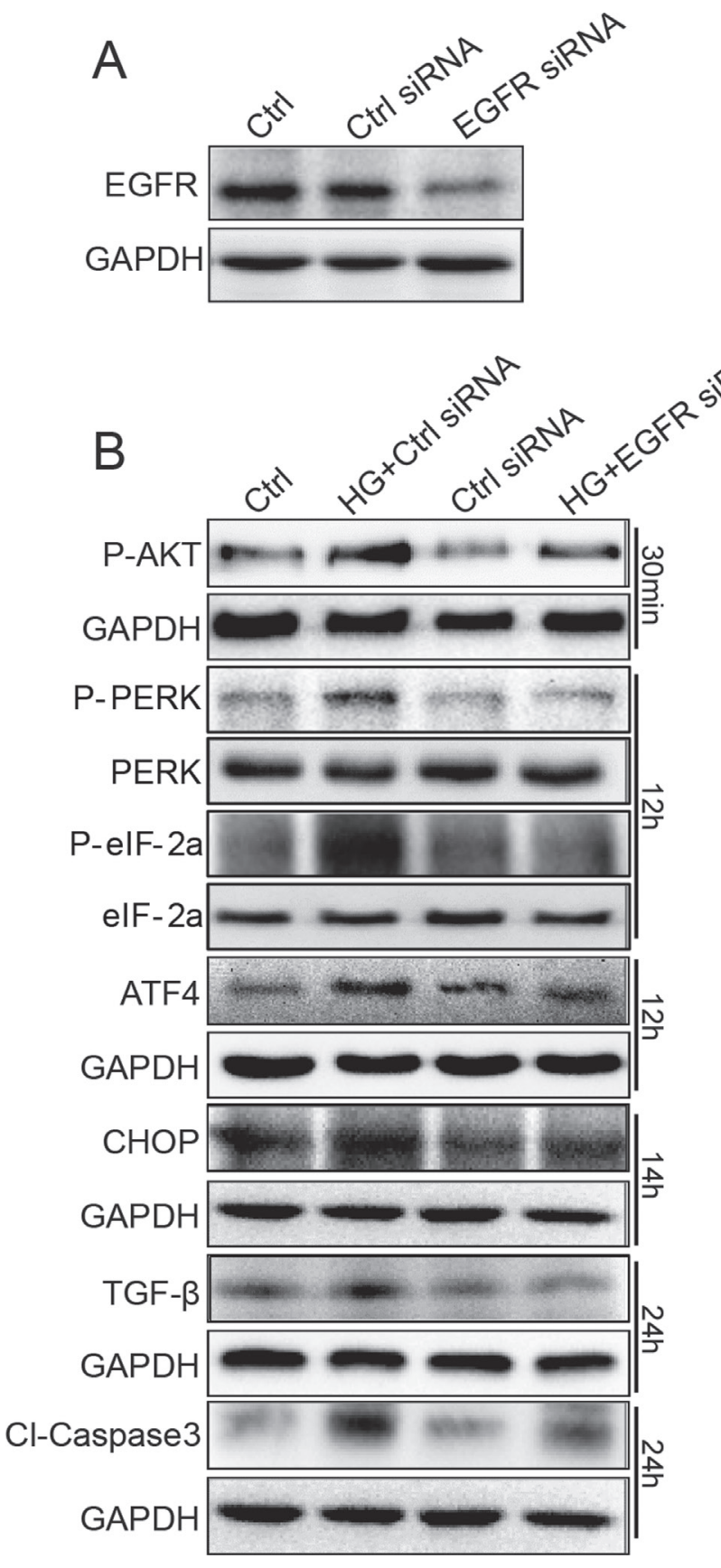

We previously discovered that EGFR has a key role in the pathogenesis cardiac damage and remodeling in STZ induced diabetic animals [9]. EGFR activation medicates renal repair after acute injury [17]. However, further exploration illustrated that persistent EGFR activation is an essential step in renal fibrosis induced by unilateral ureteral obstruction [18], subtotal nephrectomy [19], renal hypertension [20], or angiotensin II/endothelin $[21,22]$ triggered renal injury. Here we provided direct

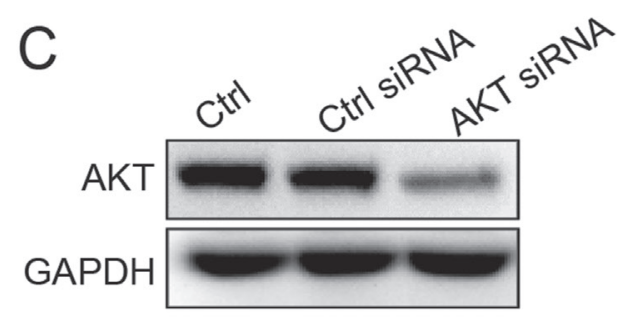

Figure 6: EGFR and AKT mediate HG-induced ER stress and cell damage in SV40 MES 13 cells. (A) SV40 cells were transfected with EGFR siRNA or control siRNA for $48 \mathrm{~h}$, the expression of EGFR was detected by Western blot analysis. (B) EGFR silencing by siRNA reduced HG-induced ER stress, fibrosis and apoptosis. (C) SV40 cells were transfected with AKT siRNA or control siRNA for $48 \mathrm{~h}$, the expression of AKT was detected by Western blot analysis. (D) AKT silencing by siRNA reduced HG-induced ER stress, fibrosis and apoptosis. Data were obtained from three independent experiments. 
evidence that suppressing EGFR prevents renal fibrosis and apoptosis in mice. These data are consistent with increased EGFR signaling leads to pathological damage in DN patients [23]. We also found that AKT knockdown also inhibited TGF- $\beta 1$ expression in renal mesangial SV40 cells, which is consistent with our previous observations in cardiomyocytes [9, 24] and kidney epithelial cells [25]. TGF- $\beta 1$ is thought to be the core factor that contributes to the renal fibrosis of DN. Recently, experimental evidence showed that TGF- $\beta 1$ participates in the activation of the $\mathrm{PI} 3 \mathrm{~K} / \mathrm{AKT}$ pathway, suggesting that AKT is a downstream mediator of TGF- $\beta 1[26,27]$. Thus, TGF- $\beta$ and AKT may positively crosstalk to regulate each other, promoting tissue fibrosis and other phenotypes.

Accumulating evidence indicates that oxidative stress and endoplasmic reticulum stress contribute to development and progression of diabetes and complications. Both increased ROS production and ER stress were observed in the current study, with similar results with other independent groups [28, 29]. In this study, we set to explore the molecular mechanisms underlying ROS/ER stressduring DN progression. As a direct target of EGFR, AKT phosphorylation is blocked by EGFR inhibition through either AG1478 or EGFR siRNA, and the phosphorylation of AKT regulates oxidative stress and ER stress in DN. Activated EGFR was reported to induce NOX activation via ERK signaling pathway, and consequently influenced the production of $\operatorname{ROS}[17,30]$. However, we found that EGFR directly caused AKT phosphorylation. Evidence has demonstrated that AKT activation positively regulates ER stress via PrPC and AktMnSOD pathway in mesenchymal stem cells [31]. Also, PI3K-Akt signal inhibition affects expression of genes related to endoplasmic reticulum stress in primary goose hepatocytes [32]. Thus, our finding that AKT knockdown prevents HG-induced protein expression involved in ER stress activation further confirmed the regulation of AKT on ER stress in renal cells. Interestingly, NAC, a scavenger of ROS, could also inhibit ER stress, suggesting that ROS accumulation could trigger ER stress. ROS is linked to ER stress in other systems, such as rat cardiac cells, and human hepatoma cells $[33,34]$. Reports on the interactions between ROS and ER stress are still controversial and not clear $[9,35,36]$. Here we provide direct evidence that inhibition of ROS could alleviate ER stress in SV40 cells.

It is known that EGFR activation is a key component in diabetic damage; it involves in insulin sensitivity [37], and leads to diabetic cardiomyopathy [9]. Chen et al.

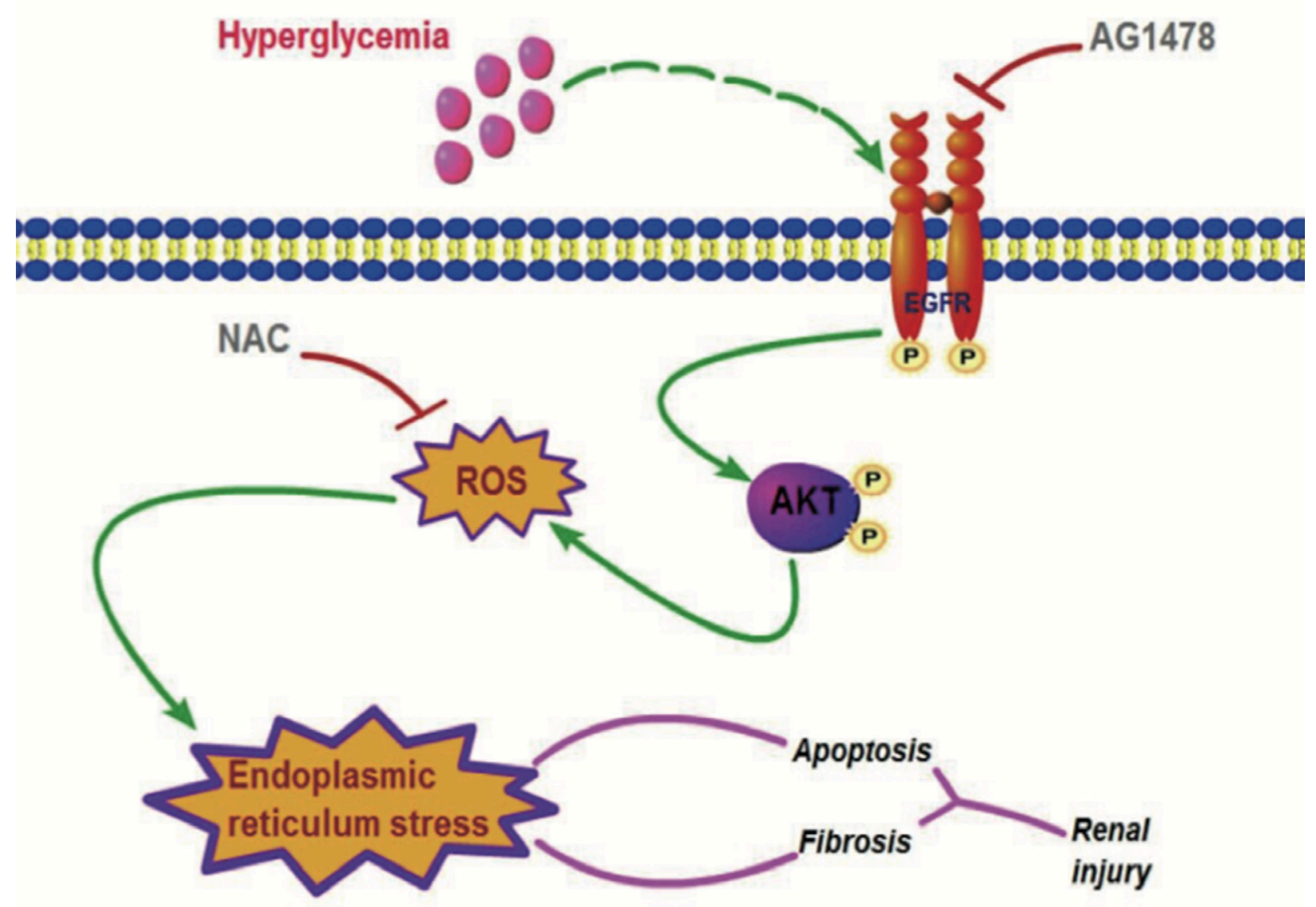

Figure 7: Scheme for EGFR/AKT/ROS/ER stress signaling pathway in preventing DN. Inhibition of EGFR by AG1478 eliminated AKT phosphorylation, sequentially reduced oxidative stress and ER stress, decreased diabetes-induced renal. 
reported Hippo pathway may be linked to EGFR-mediated renal epithelial injury in diabetes, however, they failed to include oxidative stress and ER stress pathway as one of the critical contributors to these pathological changes [38]. Oxidative stress is well characterized in cardiovascular disease, and ROS trans-activates EGFR through HBEGF production [39]. Our study confirmed the crosstalk between ROS accumulation and EGFR transactivation in renal system, which has been reported to mediate various cellular responses in inflammation, fibrosis, and apoptosis $[40,41]$.

Using knock-down approach to inhibit EGFR and AKT, we successfully rescued ROS/ ER stress mediated apoptosis and fibrosis, further illustrated that EGFR/ AKT pathway plays a pivot role in the progress of DN. Future investigation will focus on confirming these molecular cascades in living animals. We expect that renal microvascular function is altered, as Akhtar's group showed that in vascular smooth muscle cells (VSMCs), inhibition of EGFR/ErbB2 reverses the vascular dysfunction. EGFR could be a potential clinical strategy in diabetic patients with microvascular complications [42]. Since EGFR signaling has various roles in cardiovascular system [43], controlling EGFR phosphorylation is important to improve the prognosis of diabetic patients.

Due to the pathogenic effects of EGFR in multiple studies, EGFR antagonism by antibodies or smallmolecule inhibitors has been shown to have beneficial effects in cancer patients [44]. EGFR monoclonal antibodies are tumor specific and react with breast and lung carcinomas and malignant gliomas [45]. EGFR antagonism using AG1478 reduces cardiac fibrosis in diabetic mice and improved metabolic status via inhibition of eIF2 $\alpha /$ ATF4 pathway [46]. Inhibition of EGFR using AG1478 is also reported to prevent progressive kidney injury through mTOR/AMPK pathway. Here we have utilized STZ-induced diabetic mice model and demonstrate that AG1478 markedly attenuates ER stress and promotes renal fibrosis through inhibition of EGFR/ AKT activation.

In conclusion, we demonstrated that EGFR plays a pivot role in diabetic nephropathy pathogenesis via upregulating ROS generation and ER stress. Inhibition of EGFR attenuates damage of DN through EGFR/AKT/ ROS/ER stress signaling, which could be a potential therapeutic target in diabetic kidney diseases.

\section{MATERIALS AND METHODS}

\section{Reagents, cell culture and treatment}

AG1478 and NAC were purchased from Sigma (St. Louis,MO). AG1478 was dissolved in DMSO for in vitro experiments and in sodium carboxyl methyl cellulose (CMC-Na) (0.5\%) for in vivo experiments. NAC was dissolved in DMSO for in vitro experiments. SV40 MES
13 mesangial cell line was obtained from the Shanghai Institute of Biochemistry and Cell Biology (Shanghai, China) and cultured in 3:1 mixture of DMEM medium (Gibco, Eggenstein, Germany) containing $5.5 \mathrm{mmol} / \mathrm{L}$ of D-glucose and Ham's F12 medium with 14mM HEPES supplemented with $10 \% \mathrm{FBS}, 100 \mathrm{U} / \mathrm{ml}$ of penicillin, and $100 \mathrm{mg} / \mathrm{mL}$ of streptomycin. In the high glucose-treated group (HG), cells were incubated with a DMEM medium containing $33 \mathrm{mmol} / \mathrm{L}$ of D-glucose.

\section{Animals}

The animals were obtained from Animal Center of Wenzhou Medical University. Protocols used for all animal studies were approved by the Wenzhou Medical University Animal Policy and Welfare Committee. All mice had free access to food and water at all times. Diabetes mellitus was induced in male C57/BL6 mice 8 to 12 weeks old, weighing 18 to $22 \mathrm{~g}$ by a single intraperitoneal (i.p.) injection of STZ (Sigma Chemicals, St. Louis, MO) at the dose of $100 \mathrm{mg} / \mathrm{kg}$ dissolved in $100 \mathrm{mM}$ citrate buffer ( $\mathrm{pH} 4.5$ ). Control animals received buffered saline alone. One week after STZ injection, blood glucose levels were measured using a Glucometer by mandibular vein puncture blood sampling. Mice with fasting-blood glucose $>12 \mathrm{mmol} / \mathrm{L}$ were considered diabetic and were used for the further study. The compound treatment was started 1 week after STZ injection. Diabetic mice were orally treated with AG1478 (AG, $20 \mathrm{mg} / \mathrm{kg}$ ), vehicle (CMC$\mathrm{Na})$ by gavage every other day for 8 weeks $(n=8$ in each group). The corresponding control groups were treated with vehicle for 8 weeks $(n=8)$. At the indicated time points, blood glucose was measured and body weight was recorded (Supplementary Figure 1). 56 days after the first treatment, mice were sacrificed under anestheisa. The body weight was recorded at the end of death and the blood samples were collected and centrifuged at $4^{\circ} \mathrm{C}$ at $3000 \mathrm{rpm}$ for $10 \mathrm{~min}$ to collect the sera. Kidney tissues were collected and embedded in 4\% paraformaldehyde for pathological analysis and/or snap-frozen in liquid nitrogen for gene and protein expression analysis.

\section{Western blot analysis}

Cell and tissue homogenates were prepared. Protein samples $(30-80 \mu \mathrm{g})$ were subjected to $10 \%$ sodium dodecyl sulfate-polyacrylamide gel electrophoresis, and transferred onto polyvinyldene fluoride membrane (Bio-Rad Laboratory, Hercules, CA). After incubating in blocking buffer (5\% milk in tris-buffered saline containing $0.05 \%$ Tween 20) for $1.5 \mathrm{~h}$ at room temperature, membranes were incubated with different primary antibodies overnight at $4{ }^{\circ} \mathrm{C}$. Antibodies for p-EGFR/EGFR, p-AKT/AKT, p-PERK/PERK, p-eIF $2 \alpha /$ eIF $2 \alpha$, Bax, GAPDH, TGF- $\beta 1$, collagen IV, CHOP, cleaved-Caspase 3 and were obtained from Santa Cruz Technology (Santa Cruz, CA), and 
antibodies for ATF4, CHOP were purchased from Abcam (Cambride, MA). Then membranes were washed in TBST and reacted with secondary horseradish peroxidaseconjugated antibody (Santa Cruz, CA; 1:5000) for $1-2 \mathrm{~h}$ at room temperature. Antigen-antibody complexes were then visualized using enhanced chemiluminescence reagents (Bio-Rad, Hercules, CA). The density of the immunoreactive bands was analyzed using Image $\mathrm{J}$ software (NIH, Bethesda, MD).

\section{Reverse transcription and real-time quantitative PCR}

Total RNA was isolated from cells and tissues (50-100 mg) using TRIZOL (Invitrogen, Carlsbad, CA) according to the manufacturer's instructions. Reverse transcription and quantitative PCR were performed using M-MLV Platinum RT-qPCR Kit (Invitrogen, Carlsbad, CA). Real-time qPCR was carried out using the Eppendorf Real plex 4 instrument (Eppendorf, Hamburg, Germany). The following primers were synthesized from Invitrogen: collagen IV, sense: TGGCCTTGGAGGAAACTTTG, and antisense: CTTGGAAACCTTGTGGACCAG; TGF- $\beta 1$, sense: TGACGTCACTGGAGTTGTACGG, and antisense: GGTTCATGTCATGGATGGTGC; and b-actin, sense: CCGTGAAAAGATGACCCAGA, and antisense: TACGACCAGAGGCATACAG. The relative amount of each gene was normalized to the amount of $\beta$-actin.

\section{Determination of ROS generation by fluorescent microscope and flow cytometry}

In order to analyze the ROS generation, a subtype of ROS such as hydrogen peroxide (H2O2) was detected using $2 \mu \mathrm{M}$ DCFH-DA, respectively, as described previously [47]. The fluorescence intensity for 10,000 events was acquired using FACS, and cellular images were captured under the Nikon fluorescence microscope (400×amplification; Nikon, Japan).

\section{Transient transfection of EGFR and AKT si- RNA}

The small interfering RNA (si-RNA) specifically targeting the nucleotides of EGFR or AKT and its control si-RNA containing negative scrambled sequences were obtained from Gene Pharma LTD. (Shanghai, China). Transfection of SV40 cells with siRNAs was carried out using LipofectAMINE ${ }^{\mathrm{TM}} 2000$ (Invitrogen, Carlsbad, CA), according to the manufacturer's instruction. After $48 \mathrm{~h}$ of transfection, the transfected cells were then treated with $\mathrm{HG}$ for the following experiments.

\section{Determination of renal superoxide production}

We evaluated renal superoxide production with in situ dihydroethidium (DHE) staining using the method described previously [47]. In brief, kidneys from mice were excised, immediately embedded in OCT compound, and cut into $5 \mu \mathrm{m}$-thick sections. The sections were incubated with DHE in PBS $(10 \mathrm{mmol} / \mathrm{L})$ in a dark and humidified container at $37^{\circ} \mathrm{C}$ for $45 \mathrm{~min}$. DHE is oxidized upon reaction with superoxide to ethidium bromide, which binds to DNA in the nucleus and fluoresces red. The images were viewed under the fluorescence microscope $(\lambda$ ex $490 \mathrm{~nm}$, $\lambda$ em610 nm, 400×amplification; Nikon, Japan).

\section{Measurement of Serum Biomarkers}

The components of serum albumin were detected using commercial kits (Nanjing Jiancheng Bioengineering Institute, Jiangsu, People's Republic of China).

\section{Histological analysis}

Kidneys were fixed in 4\% paraformaldehyde solution, embedded in paraffin, and sectioned at $5 \mu \mathrm{m}$. After dehydration, sections were stained with hematoxylin and eosin (H\&E), masson's trichrome, sirius red, respectively, according to the previously reported methods [48]. The stained sections were then viewed under the fluorescence microscope (400×amplification; Nikon, Japan).

\section{Immunohistochemistry analysis}

Kidneys were fixed in $4 \%$ paraformaldehyde solution, embedded in paraffin, and sectioned at $5 \mu \mathrm{m}$. After dehydration, sections were subjected to antigen retrieval in $0.01 \mathrm{~mol} / \mathrm{L}$ citrate buffer $(\mathrm{pH} 6.0)$ by microwaving, and then placed in 3\% hydrogen peroxide in methanol for $30 \mathrm{~min}$ at room temperature. After blocking with $5 \%$ BSA, the sections were incubated with anti-p-EGFR antibody (1:300, Santa Cruz, CA, USA), anti-p-AKT antibody (1:300, Santa Cruz, CA, USA), anti-3-NT antibody (1:500, Abcam Inc, MA), anti-ATF-4 antibody (1:500, Abcam Inc, MA), or antiCHOP antibody(1:500, Abcam Inc, MA), respectively, overnight ant $4{ }^{\circ} \mathrm{C}$, followed by the appropriate secondary antibody (1:200, Santa Cruz, CA, USA). The reaction was visualized with DAB solution. After counterstaining with hematoxylin, the sections were dehydrated and viewed under a light microscope $(400 \times$ amplification; Nikon, Japan).

\section{TUNEL staining}

Kidney tissue sections of $5 \mu \mathrm{m}$ were used for the terminal deoxynucleotidyl transferase-mediated dUTP nick end labeling (TUNEL) apoptosis detection kit (R\&D Systems, Minneapolis, MN) according to the manufacturer's instruction. TUNEL positive cells were imaged under a fluorescence microscope (400×amplification; Nikon Tokyo, Japan). 


\section{Statistical analysis}

Results are expressed as mean \pm SDs of three independent experiments for the in vitro studies or of 7 mice for the in vivo experiments. The statistical significance of differences between groups was obtained by the student's $t$-test or ANOVA multiple comparisons in GraphPad Pro5.0 (Graphpad, San Diego, CA). Differences were considered to be significant at $* P<0.05 ; * * P<0.01$; $* * * P<0.005$.

\section{Authors' contributions}

Z.X., Q.W., Y.Q., P.Z., and J.H. researched data; G.L., P.Z., C.Z., and Y.Z. contributed initial discussion of the project; Y.Z. and C.Z. reviewed the article; G.L., J.W., and Z.X. wrote the article.

\section{ACKNOWLEDGMENTS}

This work was supported by the National Natural Science Foundation of China (81622043, 21572166, 81500657, and 81570347), and Natural Science Funding of Zhejiang Province (LR16H310001, LY16H310013 and LQ16H160019), and Science and Technology Grant of Wenzhou City (Y20150085).

\section{CONFLICTS OF INTEREST}

The authors declare no conflicts of interest.

\section{REFERENCES}

1. Kanwar YS, Wada J, Sun L, Xie P, Wallner EI, Chen S, Chugh S, Danesh FR. Diabetic nephropathy: mechanisms of renal disease progression. Exp Biol Med (Maywood). 2008; 233:4-11.

2. Parchwani DN, Upadhyah AA. Diabetic nephropathy: Progression and pathophysiology. Int J Med Sci Public Health. 2012; 1:59-70.

3. Sen U, Pushpakumar S. Mini-review: diabetic renal complications, a potential stinky remedy. Am J Physiol Renal Physiol. 2016; 310:F119-122.

4. Khan SS, Quaggin SE. Therapies on the Horizon for Diabetic Kidney Disease. Curr Diab Rep. 2015; 15:111.

5. Soetikno V, Arozal W, Louisa M, Setiabudy R. New insight into the molecular drug target of diabetic nephropathy. Int J Endocrinol. 2014; 2014:968681.

6. Schena FP, Gesualdo L. Pathogenetic mechanisms of diabetic nephropathy. J Am Soc Nephrol. 2005; 16:S30-33.

7. Panchapakesan U, Pollock C, Saad S. Renal epidermal growth factor receptor: its role in sodium and water homeostasis in diabetic nephropathy. Clin Exp Pharmacol Physiol. 2011; 38:84-88.
8. Zhang MZ, Wang Y, Paueksakon P, Harris RC. Epidermal growth factor receptor inhibition slows progression of diabetic nephropathy in association with a decrease in endoplasmic reticulum stress and an increase in autophagy. Diabetes. 2014; 63:2063-2072.

9. Liang D, Zhong P, Hu J, Lin F, Qian Y, Xu Z, Wang J, Zeng C, Li X, Liang G. EGFR inhibition protects cardiac damage and remodeling through attenuating oxidative stress in STZinduced diabetic mouse model. J Mol Cell Cardiol. 2015; 82:63-74.

10. Cunard R. Endoplasmic Reticulum Stress in the Diabetic Kidney, the Good, the Bad and the Ugly. J Clin Med. 2015; 4:715-740.

11. Zeeshan H, Lee G, Kim HR, Chae HJ. Endoplasmic Reticulum Stress and Associated ROS. Int J Mol Sci. 2016; 17:327.

12. Malhotra JD, Kaufman RJ. Endoplasmic reticulum stress and oxidative stress: a vicious cycle or a double-edged sword? Antioxid Redox Signal. 2007; 9:2277-2293.

13. Simon HU, Haj-Yehia A, Levi-Schaffer F. Role of reactive oxygen species (ROS) in apoptosis induction. Apoptosis. 2000; 5:415-418.

14. Kim HS, Lim JM, Kim JY, Kim Y, Park S, Sohn J. Panaxydol, a component of Panax ginseng, induces apoptosis in cancer cells through EGFR activation and ER stress and inhibits tumor growth in mouse models. Int $\mathrm{J}$ Cancer. 2016; 138:1432-1441.

15. Sugita $\mathrm{S}$, Ito $\mathrm{K}$, Yamashiro $\mathrm{Y}$, Moriya $\mathrm{S}$, Che $\mathrm{XF}$, Yokoyama T, Hiramoto M, Miyazawa K. EGFR-independent autophagy induction with gefitinib and enhancement of its cytotoxic effect by targeting autophagy with clarithromycin in non-small cell lung cancer cells. Biochem Biophys Res Commun. 2015; 461:28-34.

16. Bienaime F, Canaud G, El Karoui K, Gallazzini M, Terzi F. Molecular pathways of chronic kidney disease progression. Nephrol Ther. 2016; 12:S35-38.

17. Dong J, Ramachandiran S, Tikoo K, Jia Z, Lau SS, Monks TJ. EGFR-independent activation of p38 MAPK and EGFR-dependent activation of ERK1/2 are required for ROS-induced renal cell death. Am J Physiol Renal Physiol. 2004; 287:F1049-1058.

18. Terzi F, Burtin M, Hekmati M, Federici P, Grimber G, Briand P, Friedlander G. Targeted expression of a dominantnegative EGF-R in the kidney reduces tubulo-interstitial lesions after renal injury. J Clin Invest. 2000; 106:225-234.

19. Liu N, Guo JK, Pang M, Tolbert E, Ponnusamy M, Gong R, Bayliss G, Dworkin LD, Yan H, Zhuang S. Genetic or pharmacologic blockade of EGFR inhibits renal fibrosis. J Am Soc Nephrol. 2012; 23:854-867.

20. Francois H, Placier S, Flamant M, Tharaux PL, Chansel D, Dussaule JC, Chatziantoniou C. Prevention of renal vascular and glomerular fibrosis by epidermal growth factor receptor inhibition. FASEB J. 2004; 18:926-928. 
21. Chansel D, Ciroldi M, Vandermeersch S, Jackson LF, Gomez AM, Henrion D, Lee DC, Coffman TM, Richard S, Dussaule JC, Tharaux PL. Heparin binding EGF is necessary for vasospastic response to endothelin. FASEB J. 2006; 20:1936-1938.

22. Chen J, Chen JK, Nagai K, Plieth D, Tan M, Lee TC, Threadgill DW, Neilson EG, Harris RC. EGFR signaling promotes TGFbeta-dependent renal fibrosis. J Am Soc Nephrol. 2012; 23:215-224.

23. Advani A, Wiggins KJ, Cox AJ, Zhang Y, Gilbert RE, Kelly DJ. Inhibition of the epidermal growth factor receptor preserves podocytes and attenuates albuminuria in experimental diabetic nephropathy. Nephrology. 2011; 16:573-581.

24. Peng K, Tian X, Qian Y, Skibba M, Zou C, Liu Z, Wang J, $\mathrm{Xu}$ Z, Li X, Liang G. Novel EGFR inhibitors attenuate cardiac hypertrophy induced by angiotensin II. J Cell Mol Med. 2016; 20:482-494.

25. Fang Q, Zou C, Zhong P, Lin F, Li W, Wang L, Zhang Y, Zheng C, Wang Y, Li X, Liang G. EGFR mediates hyperlipidemia-induced renal injury via regulating inflammation and oxidative stress: the detrimental role and mechanism of EGFR activation. Oncotarget. 2016; 7:24361-24373. doi: 10.18632/oncotarget.8222.

26. Li J, Ren J, Liu X, Jiang L, He W, Yuan W, Yang J, Dai C. Rictor/mTORC2 signaling mediates TGFbeta1-induced fibroblast activation and kidney fibrosis. Kidney Int. 2015; 88:515-527.

27. Park JT, Kato M, Yuan H, Castro N, Lanting L, Wang M, Natarajan R. FOG2 protein down-regulation by transforming growth factor-betal-induced microRNA200b/c leads to Akt kinase activation and glomerular mesangial hypertrophy related to diabetic nephropathy. J Biol Chem. 2013; 288:22469-22480.

28. Lee ES, Kim HM, Kang JS, Lee EY, Yadav D, Kwon MH, Kim YM, Kim HS, Chung $\mathrm{CH}$. Oleanolic acid and $\mathrm{N}$-acetylcysteine ameliorate diabetic nephropathy through reduction of oxidative stress and endoplasmic reticulum stress in a type 2 diabetic rat model. Nephrol Dial Transplant. 2016; 31:391-400.

29. Takayanagi T, Kawai T, Forrester SJ, Obama T, Tsuji T, Fukuda Y, Elliott KJ, Tilley DG, Davisson RL, Park JY, Eguchi S. Role of epidermal growth factor receptor and endoplasmic reticulum stress in vascular remodeling induced by angiotensin II. Hypertension. 2015; 65:1349-1355.

30. Sancho P, Bertran E, Caja L, Carmona-Cuenca I, Murillo MM, Fabregat I. The inhibition of the epidermal growth factor (EGF) pathway enhances TGF-beta-induced apoptosis in rat hepatoma cells through inducing oxidative stress coincident with a change in the expression pattern of the NADPH oxidases (NOX) isoforms. Biochim Biophys Acta. 2009; 1793:253-263.

31. Yoon YM, Lee JH, Yun SP, Han YS, Yun CW, Lee HJ, Noh H, Lee SJ, Han HJ, Lee SH. Tauroursodeoxycholic acid reduces ER stress by regulating of Akt-dependent cellular prion protein. Sci Rep. 2016; 6:39838.

32. Song Q, Han CC, Xiong XP, He F, Gan W, Wei SH, Liu HH, Li L, Xu HY. PI3K-Akt-mTOR signal inhibition affects expression of genes related to endoplasmic reticulum stress. Genet Mol Res. 2016; 15.

33. Wang CL, Liu C, Niu LL, Wang LR, Hou LH, Cao XH. Surfactin-induced apoptosis through ROS-ERS-Ca2+-ERK pathways in HepG2 cells. Cell Biochem Biophys. 2013; 67:1433-1439.

34. Zhang Q, Li H, Wang S, Liu M, Feng Y, Wang X. Icariin protects rat cardiac $\mathrm{H} 9 \mathrm{c} 2$ cells from apoptosis by inhibiting endoplasmic reticulum stress. Int J Mol Sci. 2013; 14:17845-17860.

35. Jang JH, Iqbal T, Min KJ, Kim S, Park JW, Son EI, Lee TJ, Kwon TK. Helenalin-induced apoptosis is dependent on production of reactive oxygen species and independent of induction of endoplasmic reticulum stress in renal cell carcinoma. Toxicol In Vitro. 2013; 27:588-596.

36. Xu C, Ding W, Yang L, Yang M, Zhang M, Gu Y. Contributions of endoplasmic reticulum stress and reactive oxygen species to renal injury in aldosterone/salt-induced rats. Nephron Exp Nephrol. 2014; 126:25-32.

37. Rogers C, Moukdar F, McGee MA, Davis B, Buehrer BM, Daniel KW, Collins S, Barakat H, Robidoux J. EGF receptor (ERBB1) abundance in adipose tissue is reduced in insulin-resistant and type 2 diabetic women. J Clin Endocrinol Metab. 2012; 97:E329-340.

38. Chen J, Harris RC. Interaction of the EGF Receptor and the Hippo Pathway in the Diabetic Kidney. J Am Soc Nephrol. 2016; 27:1689-1700.

39. Frank GD, Mifune $M$, Inagami $T$, Ohba $M$, Sasaki $T$, Higashiyama S, Dempsey PJ, Eguchi S. Distinct mechanisms of receptor and nonreceptor tyrosine kinase activation by reactive oxygen species in vascular smooth muscle cells: role of metalloprotease and protein kinase C-delta. Mol Cell Biol. 2003; 23:1581-1589.

40. Higuchi S, Ohtsu H, Suzuki H, Shirai H, Frank GD, Eguchi S. Angiotensin II signal transduction through the AT1 receptor: novel insights into mechanisms and pathophysiology. Clin Sci (Lond). 2007; 112:417-428.

41. Schreier B, Gekle M, Grossmann C. Role of epidermal growth factor receptor in vascular structure and function. Curr Opin Nephrol Hypertens. 2014; 23:113-121.

42. Benter IF, Sarkhou F, Al-Khaldi AT, Chandrasekhar B, Attur S, Dhaunsi GS, Yousif MH, Akhtar S. The dual targeting of EGFR and ErbB2 with the inhibitor Lapatinib corrects high glucose-induced apoptosis and vascular dysfunction by opposing multiple diabetes-induced signaling changes. J Drug Target. 2015; 23:506-518.

43. Forrester SJ, Kawai T, O’Brien S, Thomas W, Harris RC, Eguchi S. Epidermal Growth Factor Receptor 
Transactivation: Mechanisms, Pathophysiology, and Potential Therapies in the Cardiovascular System. Annu Rev Pharmacol Toxicol. 2016; 56:627-653.

44. Wieduwilt MJ, Moasser MM. The epidermal growth factor receptor family: biology driving targeted therapeutics. Cell Mol Life Sci. 2008; 65:1566-1584.

45. Wikstrand CJ, Hale LP, Batra SK, Hill ML, Humphrey PA, Kurpad SN, McLendon RE, Moscatello D, Pegram CN, Reist CJ. Monoclonal antibodies against EGFRvIII are tumor specific and react with breast and lung carcinomas and malignant gliomas. Cancer Res. 1995; 55:3140-3148.

46. Galan M, Kassan M, Choi SK, Partyka M, Trebak M, Henrion D, Matrougui K. A novel role for epidermal growth factor receptor tyrosine kinase and its downstream endoplasmic reticulum stress in cardiac damage and microvascular dysfunction in type 1 diabetes mellitus. Hypertension. 2012; 60:71-80.

47. Kumar S, Kain V, Sitasawad SL. Cardiotoxicity of calmidazolium chloride is attributed to calcium aggravation, oxidative and nitrosative stress, and apoptosis. Free Radic Biol Med. 2009; 47:699-709.

48. Han J, Zou C, Mei L, Zhang Y, Qian Y, You S, Pan Y, Xu Z, Bai B, Huang W, Liang G. MD2 mediates angiotensin IIinduced cardiac inflammation and remodeling via directly binding to Ang II and activating TLR4/NF-kappaB signaling pathway. Basic Res Cardiol. 2017; 112:9. 